\title{
Associações entre déficits cognitivos e qualidade de vida na esquizofrenia
}

\section{Associations between cognitive impairment and quality of life in schizophrenia}

\author{
Breno Fiuza CruZ1, João Vinícius Salgado², Fábio Lopes Rocha ${ }^{3}$ \\ 1 Psiquiatra, mestrando do curso de Pós-Graduação em Ciências da Saúde do Instituto de Previdência dos Servidores do Estado de Minas Gerais (IPSEMG). \\ 2 Programa de Pós-Graduação em Ciências da Saúde do IPSEMG. \\ 3 Programa de Pós-Graduação em Ciências da Saúde do IPSEMG e Residência em Psiquiatria do IPSEMG.
}

Recebido: 29/1/2009 - Aceito: 13/8/2009

\begin{abstract}
Resumo
Contexto: A Qualidade de Vida (QV) constitui valiosa forma de avaliação do impacto de doenças na vida diária e favorece intervenções mais efetivas sob uma perspectiva biopsicossocial. Objetivos: Este artigo procura analisar criticamente os dados disponíveis sobre as relações entre QV e cognição na esquizofrenia. Métodos: Foi realizada uma pesquisa na base de dados PubMed e Lilacs com os descritores de assunto "schizophrenia" e "cognition disorder" ou "cognition" e "quality of life" ou "outcome assessment". Resultados: Dos 27 artigos selecionados que estudavam a relação entre cognição e QV medida por meio de escalas, 20 mostraram associações. Essas foram mais importantes nos estudos que utilizaram escalas de QV administrada por pesquisador, em comparação com escalas de autoavaliação. Conclusão: A associação entre cognição e QV é mais evidente nos estudos que utilizaram medidas de QV feitas por pesquisadores do que naqueles que utilizaram autoavaliações. A avaliação cognitiva é importante não somente como parâmetro determinante de QV dos pacientes, mas também auxilia na interpretação das escalas de QV, sendo as autoavaliações mais adequadas em pacientes com menor prejuízo cognitivo.
\end{abstract}

Cruz BF, et al. / Rev Psiq Clín. 2010;37(5):233-9

Palavras-chave: Esquizofrenia, déficits cognitivos, qualidade de vida.

\begin{abstract}
Background: The Quality of Life (QoL) is a valuable way of assessing the impact of diseases in daily life and favors more effective interventions under a biopsychosocial perspective. Objectives: This article aimed to critically analyze the available data on the relationship between QoL and cognitive function in schizophrenia. Methods: We performed a search in PubMed and Lilacs databases with the terms "schizophrenia" and "cognition disorder" or "cognition" and "quality of life" or "outcome assessment". Results: From the 27 selected articles that studied the relationship between cognition and QoL measured by scales, 20 articles showed associations. The association was more robust in studies using scales administered by a researcher, compared to self-report scales. Discussion: The cognitive assessment is important not only as a parameter for determining QoL of patients, but also as a factor to be taken into account when analyzing the data obtained by self-report QoL scales.
\end{abstract}

Cruz BF, et al. / Rev Psiq Clín. 2010;37(5):233-9

Keywords: Schizophrenia, cognitive impairment, quality of life.

\section{Introdução}

A esquizofrenia é um transtorno psiquiátrico caracterizado por prejuízo cognitivo generalizado associado a déficits mais proeminentes em atenção sustentada, aprendizagem e memória verbal, memória operativa, funções executivas e linguagem ${ }^{1}$. O prejuízo cognitivo é geralmente estável ao longo do tempo e não depende da presença de sintomas positivos ou negativos, mas se relaciona ao prejuízo funcional característico da doença ${ }^{1}$. Quando se busca a reabilitação dos pacientes, portanto, é fundamental considerar os déficits cognitivos e tentar compreender como eles podem alterar o quotidiano dos pacientes.

Nesse contexto, o conceito de Qualidade de Vida (QV) é de grande importância, pois constitui valiosa forma de avaliação do impacto de doenças na vida diária. Um dos aspectos interessantes da QV é que ela traz um componente subjetivo que abrange as percepções do indivíduo sobre si e sobre os prejuízos que a doença lhe causa. Essa característica favorece intervenções mais efetivas sob uma perspectiva biopsicossocial, além da simples redução de sintomas ${ }^{2}$. Assim, medidas de QV estão sendo cada vez mais usadas na avaliação de desempenho funcional, ensaios com drogas, aprovação de novos medicamentos, avaliação de programas de reabilitação e alocação de recursos³ ${ }^{3}$.
A QV, contudo, é um conceito amplo, que não possui uma definição precisa. Apesar dos esforços em encontrar definições e em desenvolver ferramentas de avaliação correspondentes, ainda não há um consenso claro a respeito de seus componentes ou de como se deve medi-la. A exploração do conceito de QV, portanto, requer foco e seleção dos vários potenciais significados do conceito, dependendo do objetivo do estudo 4 .

A principal forma de se avaliar a QV em transtornos psiquiátricos é por meio de escalas ou questionários. Essas escalas podem ser globais ou multidimensionais, genéricas ou específicas para a doença estudada e autoadministradas ou administradas por um entrevistador ${ }^{3}$.

Desde os anos 1980, a QV tem sido frequentemente usada como critério de resultado em pesquisa na esquizofrenia ${ }^{5}$. O número de trabalhos que estudaram a relação entre déficits cognitivos e QV na esquizofrenia vem crescendo nos últimos anos. Há carência, entretanto, de estudos que analisem criticamente o conjunto dos resultados desses trabalhos.

Este estudo tem com objetivo analisar criticamente os dados disponíveis sobre as relações entre QV e funções cognitivas na esquizofrenia. 


\section{Métodos}

Foi realizada revisão sobre o tema nas bases de dados PubMed e Lilacs, utilizando os descritores de assunto (MeSH) "schizophrenia" e "cognition disorder" e "quality of life". Também foram feitas buscas, substituindo-se "quality of life" por "outcome assessment" e/ou "cognition disorder" por "cognition", com o objetivo de ampliar a quantidade de artigos analisados. A revisão incluiu artigos publicados até outubro de 2008 nos idiomas inglês, espanhol e português.

\section{Resultados}

A busca resultou em 356 artigos. Foram excluídos os artigos que, embora contivessem avaliações como desempenho social, atividades de vida diária e nível de vida independente, não continham avaliações de qualidade de vida realizadas por meio de escalas específicas e validadas para esse fim. Com a finalidade de homogeneizar a amostra, também foram excluídos os artigos cuja avaliação de QV estava relacionada a ensaios farmacológicos ou faziam parte da avaliação de programas de reabilitação. Assim, foram selecionados 27 artigos, os quais apresentavam escalas específicas para avaliação de QV e a utilização de alguma avaliação neuropsicológica ou cognitiva como preditora da variação na QV. A tabela 1 resume algumas características dos artigos selecionados.

\section{Características gerais dos estudos e seus instrumentos}

A maioria dos trabalhos foi realizada após o ano 2000. Todos são estudos seccionais (transversais), com exceção do estudo de Sota e Heinrichs (2004)16, Addington e Addington (2008)26 e Perlick et al. $(2008)^{29}$.

Tabela 1. Características dos artigos analisados

\begin{tabular}{|c|c|c|c|c|c|}
\hline Estudo & População & Funções cognitivas & Medidas QV & Resultados & Outras associações \\
\hline Meltzer et al. (1996)6 & $\begin{array}{l}174 \text { pacientes testados } \\
\text { anteriormente a } \\
\text { tratamento, a maioria sem } \\
\text { uso de antipsicóticos }{ }^{*}\end{array}$ & $\begin{array}{l}\text { Função executiva, } \\
\text { velocidade de } \\
\text { processamento, memória } \\
\text { verbal e fluência verbal }\end{array}$ & Quality of Life Scale (OLS) & $\begin{array}{l}\text { Associações entre OLS e } \\
\text { função executiva, velocidade } \\
\text { de processamento e } \\
\text { memória verbal }\end{array}$ & $\begin{array}{l}\text { O estudo não analisou } \\
\text { influência de sintomas } \\
\text { sobre a OLS }\end{array}$ \\
\hline Heslegrave et al. (1997)2 & $\begin{array}{l}42 \text { pacientes estáveis e } \\
\text { crônicos }\end{array}$ & $\begin{array}{l}\text { Velocidade psicomotora, } \\
\text { percepção visual, função } \\
\text { executiva, memória } \\
\text { sensorial }\end{array}$ & $\begin{array}{l}\text { Sickness Impact Profile } \\
\text { (SIP)* }\end{array}$ & $\begin{array}{l}\text { Associação não } \\
\text { significativa ou muito } \\
\text { baixa }\end{array}$ & $\begin{array}{l}\text { Escala Global PANSS } \\
>\text { sintomas negativos > } \\
\text { sintomas positivos }\end{array}$ \\
\hline Dickerson et al. (1998)7 & $\begin{array}{l}72 \text { pacientes estáveis } \\
\text { vivendo na comunidade }\end{array}$ & $\begin{array}{l}\text { Habilidade verbal, } \\
\text { memória de trabalho } \\
\text { e velocidade de } \\
\text { processamento, função } \\
\text { executiva, habilidade } \\
\text { visioconstrutora, } \\
\text { memória visual, atenção, } \\
\text { velocidade psicomotora } \\
\end{array}$ & $\begin{array}{l}\text { Quality of Life Interview } \\
\text { (Q0LI)+ }\end{array}$ & Não houve associação & $\begin{array}{l}\text { Sintomas depressivos } \\
\text { e número de visitas ao } \\
\text { psiquiatra }\end{array}$ \\
\hline $\begin{array}{l}\text { Addington e Addington } \\
(1999)^{8}\end{array}$ & $\begin{array}{l}80 \text { pacientes vivendo na } \\
\text { comunidade }\end{array}$ & $\begin{array}{l}\text { Habilidade verbal, } \\
\text { atenção visual, memória } \\
\text { verbal e visual, habilidade } \\
\text { visioconstrutora e função } \\
\text { executiva }\end{array}$ & QLS\$ & $\begin{array}{l}\text { Função executiva foi } \\
\text { relacionada ao escore } \\
\text { global da OLS** }\end{array}$ & $\begin{array}{l}\text { Sintomas negativos e } \\
\text { positivos }\end{array}$ \\
\hline
\end{tabular}

* Este artigo apenas cita os resultados obtidos, não descrevendo detalhes sobre sujeitos e metodologia. Após avaliação inicial, os sujeitos do estudo fizerem uso por seis meses de clozapina. \# Escore da escala dado pelos próprios pacientes

+ Escore da escala dado pelos próprios pacientes. Utilizaram-se também a SFS e a Multnomah Community Ability Scale (MCAS).

$\S 0$ estudo utilizou também The Social Functioning Scale (SFS) e The Assessment of Interpersonal Problem Solving Skills como medidas de funcionamento social.

** Quando sintomas negativos são levados em consideração, a associação deixa de ser significativa.

\begin{tabular}{|c|c|c|c|c|c|}
\hline Smith et al. (1999)9 & $\begin{array}{l}46 \text { pacientes com } \\
\text { internação no último mês }\end{array}$ & $\begin{array}{l}\text { Velocidade de } \\
\text { processamento de } \\
\text { informações, memória, } \\
\text { função executiva, } \\
\text { memória de trabalho }\end{array}$ & QOLI* & Não houve correlações & $\begin{array}{l}\text { Sintomas depressivos e } \\
\text { negativos }\end{array}$ \\
\hline Kasckow et al. (2001)10 & $\begin{array}{l}54 \text { pacientes estáveis } \\
\text { maiores de } 45 \text { anos } \\
\text { hospitalizados por pelo } \\
\text { menos } 6 \text { meses durante } 0 \\
\text { ano anterior à pesquisa* }\end{array}$ & $\begin{array}{l}\text { Miniexame do Estado } \\
\text { Mental (MEEM) }\end{array}$ & $\begin{array}{l}\text { Quality of Well-Being } \\
\text { Scale (OWB) }\end{array}$ & $\begin{array}{l}\text { Mini-Mental não se } \\
\text { associou a OWB+ }\end{array}$ & $\begin{array}{l}\text { Sintomas positivos e } \\
\text { negativos }\end{array}$ \\
\hline Aksaray et al. (2002)11 & $\begin{array}{l}57 \text { pacientes estáveis } \\
\text { vivendo na comunidade }\end{array}$ & Função executiva & $\begin{array}{l}\text { PCASSE (physical, } \\
\text { cognitive, affective, } \\
\text { social, economic-social, } \\
\text { ego functions)§ }\end{array}$ & $\begin{array}{l}\text { Associação fraca entre } \\
\text { déficits cognitivos e } 00 \mathrm{~L}\end{array}$ & $\begin{array}{l}\text { Sintomas negativos e } \\
\text { efeitos colaterais }\end{array}$ \\
\hline Vaz e Heinrichs (2002) ${ }^{12}$ & $\begin{array}{l}32 \text { pacientes estáveis } \\
\text { recebendo antipsicóticos } \\
\text { típicos** }\end{array}$ & Memória verbal & SIP & $\begin{array}{l}\text { Grupo com déficit de } \\
\text { memória apresenta pior } \\
\text { escore global na SIP geral } \\
\text { e nas subescalas de sono } \\
\text { e repouso e satisfação } \\
\text { com relações sociais }\end{array}$ & $\begin{array}{l}0 \text { estudo não analisou } \\
\text { influência de sintomas } \\
\text { sobre a SIP }\end{array}$ \\
\hline
\end{tabular}

* Foram utilizados apenas três escores subjetivos da escala.

\# Esses pacientes foram pareados com 54 pacientes de mesma faixa etária vivendo na comunidade.

+ 0 escore do Mini-Mental no início da pesquisa é preditor do escore da OWB após seis meses de seguimento.

$\S$ Escore da escala dado pelos próprios pacientes.

** Os pacientes foram divididos em 16 pacientes com déficit de memória e 16 pacientes sem déficit de memória. 
Tabela 1. Características dos artigos analisados (continuação)

\begin{tabular}{|c|c|c|c|c|c|}
\hline Estudo & População & Funções cognitivas & Medidas QV & Resultados & Outras associações \\
\hline Fujii et al. (2004)13 & $\begin{array}{l}30 \text { pacientes estáveis } \\
\text { vivendo na comunidade } \\
\text { selecionados por meio de } \\
\text { base de dados de estudos } \\
\text { anteriores, com média de } \\
15 \text { anos entre avaliação } \\
\text { cognitiva e da qualidade } \\
\text { de vida* }\end{array}$ & $\begin{array}{l}\text { Função executiva, } \\
\text { memória de trabalho, } \\
\text { memória e habilidade } \\
\text { psicomotora }\end{array}$ & $\begin{array}{l}\text { Brief Quality of Life } \\
\text { Inventory (BOOLI) - utiliza } \\
\text { medidas subjetivas e } \\
\text { objetivas } \#\end{array}$ & $\begin{array}{l}\text { Associações entre QV } \\
\text { e todas as funções } \\
\text { cognitivas estudadas+ }\end{array}$ & $\begin{array}{l}0 \text { artigo não estudou } \\
\text { outras associações }\end{array}$ \\
\hline Alptekin et al. (2004) & $\begin{array}{l}38 \text { pacientes estáveis, } \\
\text { crônicos, utilizando } \\
\text { antipsicóticos atípicos } \\
\text { (exceto 2) }\end{array}$ & $\begin{array}{l}\text { Atenção, memória de } \\
\text { trabalho, fluência verbal e } \\
\text { função executiva }\end{array}$ & $\begin{array}{l}\text { World Health Organization } \\
\text { Quality of Life - Breif } \\
\text { Form (WHOOOL-BREF)** }\end{array}$ & $\begin{array}{l}\text { Associação significativa } \\
\text { entre as duas medidas } \\
\text { cognitivas e QV }\end{array}$ & $\begin{array}{l}\text { Sem associação com } \\
\text { sintomas negativos, } \\
\text { positivos ou efeitos } \\
\text { colaterais da medicação }\end{array}$ \\
\hline Herman (2004)15 & $\begin{array}{l}43 \text { pacientes sem abuso } \\
\text { de substâncias e } 46 \\
\text { pacientes com abuso de } \\
\text { substâncias associadas, } \\
\text { em internação hospitalar }\end{array}$ & $\begin{array}{l}\text { Função executiva, função } \\
\text { intelectual e memória }\end{array}$ & WHOOOL-BREF & $\begin{array}{l}\text { Função executiva } \\
\text { (COWAT) foi o preditor } \\
\text { mais fortemente } \\
\text { relacionado à OV }\end{array}$ & $\begin{array}{l}\text { Escore total BPRS não foi } \\
\text { significativo no modelo de } \\
\text { regressão }\end{array}$ \\
\hline Sota e Heinrichs (2004)16 & $\begin{array}{l}55 \text { pacientes crônicos } \\
\text { recebendo antipsicóticos } \\
\text { típicos }{ }^{\# \#}\end{array}$ & $\begin{array}{l}\text { Inteligência, memória } \\
\text { verbal, função executiva, } \\
\text { velocidade motora e } \\
\text { destreza manual }\end{array}$ & SIP & $\begin{array}{l}\text { Inteligência, função } \\
\text { executiva, memória e } \\
\text { destreza motora bilateral } \\
\text { predizem QV }\end{array}$ & $\begin{array}{l}\text { Sintomas não foram } \\
\text { preditores significativos } \\
\text { de OV }\end{array}$ \\
\hline
\end{tabular}

* 11 pacientes com esquizofrenia paranoide, 3 com esquizofrenia indiferenciada, 1 com transtorno psicótico não especificado, 9 esquizoafetivos, 6 com transtorno bipolar.

\# Escore da escala dado pelos próprios pacientes.

+ Associações com medidas subjetivas: memória de trabalho - relações sociais; habilidade psicomotora - contato familiar; memória - atividades diárias e saúde. Associações com medidas objetivas: memória - dinheiro gasto consigo mesmo; memória de trabalho - vitimização; função executiva - contato familiar e adequação financeira.

$\S 0$ estudo utilizou 31 indivíduos saudáveis como grupo-controle.

** Escore da escala dado pelos próprios pacientes.

\# Estudo longitudinal.

\begin{tabular}{|c|c|c|c|c|c|}
\hline $\begin{array}{l}\text { Cavieres e Valdebenito } \\
(2005)^{17}\end{array}$ & $\begin{array}{l}50 \text { pacientes estáveis em } \\
\text { tratamento ambulatorial }\end{array}$ & $\begin{array}{l}\text { Atenção, função } \\
\text { executiva, memória, } \\
\text { memória de trabalho }\end{array}$ & OLS & $\begin{array}{l}\text { Atenção, função executiva } \\
\text { e memória }\end{array}$ & Sintomas negativos \\
\hline Hofer et al. (2005)18 & $\begin{array}{l}60 \text { pacientes estáveis } \\
\text { vivendo na comunidade e } \\
\text { duração da doença maior } \\
\text { que dois anos, recebendo } \\
\text { olanzapina e clozapina }\end{array}$ & $\begin{array}{l}\text { Inteligência pré-mórbida, } \\
\text { função executiva, fluência } \\
\text { verbal, aprendizado } \\
\text { verbal, memória verbal } \\
\text { e visual, memória de } \\
\text { trabalho, atenção }\end{array}$ & WHOOOL-BREF* & $\begin{array}{l}\text { Não houve associação } \\
\text { estatisticamente } \\
\text { significativa entre 00L e } \\
\text { variáveis neurocognitivas }{ }^{\#}\end{array}$ & $\begin{array}{l}0 \text { estudo não analisou } \\
\text { outras associações }\end{array}$ \\
\hline Wegener et al. (2005)19 & $\begin{array}{l}51 \text { pacientes+ em primeiro } \\
\text { surto psicótico, } 33 \\
\text { utilizando antipsicóticos } \\
\text { atípicos e } 18 \text { sem } \\
\text { medicação }\end{array}$ & $\begin{array}{l}\text { Habilidade verbal, } \\
\text { memória visual, memória } \\
\text { verbal, atenção, fluência } \\
\text { verbal e função executiva }\end{array}$ & WHOQOL-BREF & $\begin{array}{l}\text { Função executiva, fluência } \\
\text { verbal e atenção explicam } \\
\text { de } 14 \% \text { a } 28 \% \text { da } \\
\text { variação na } 0 \text { § }\end{array}$ & $\begin{array}{l}\text { Depressão, escala } \\
\text { psicopatologia geral } \\
\text { PANSS e sintomas } \\
\text { negativos explicam 43\% } \\
\text { na variação da OV }\end{array}$ \\
\hline Bozikas et al. (2006)20 & $\begin{array}{l}40 \text { pacientes crônicos } \\
\text { vivendo na comunidade }\end{array}$ & $\begin{array}{l}\text { Função executiva, fluência } \\
\text { verbal, memória de } \\
\text { trabalho, memória verbal } \\
\text { e visual, atenção auditiva } \\
\text { e visual, habilidade } \\
\text { visioespacial, velocidade } \\
\text { psicomotora }\end{array}$ & OLS & $\begin{array}{l}\text { OLS correlacionou-se } \\
\text { a fluência e memória } \\
\text { visual }^{* *}\end{array}$ & $\begin{array}{l}\text { Sintomas negativos e } \\
\text { efeitos colaterais }\end{array}$ \\
\hline
\end{tabular}

* Foram também avaliados necessidade de cuidado (Camberwell Assessment of Need - CAN) e funcionamento social (emprego e situação de vida).

\# Memória de trabalho e memória visual associaram-se a funcionamento ocupacional e vida independente.

+ Pacientes jovens (15-27 anos). Inclui diagnósticos outros que não esquizofrenia (8 TAB, 3 psicose induzida por drogas, 2 transtorno psicótico não especificado e 1 transtorno psicótico breve).

$\S$ Na presença de sintomatologia, habilidade verbal, fluência verbal e função executiva mantêm-se preditoras do fator ambiental da escala de QV, e o fator social da escala de QV mantém-se associado à fluência verbal e à função executiva.

** Testes de fluência relacionaram-se com subescala de relacionamentos interpessoais e suporte intrapsíquico. Memória visual relacionou-se com a subescala de objetos comuns e atividades. Testes de fluência também se relacionaram ao escore total OLS.

\begin{tabular}{|c|c|c|c|c|c|}
\hline Mittal et al. (2006) ${ }^{21}$ & $\begin{array}{l}199 \text { pacientes com idade } \\
\text { entre } 45 \text { e } 85 \text { anos, } \\
\text { estáveis }\end{array}$ & $\begin{array}{l}\text { Atenção, memória, função } \\
\text { executiva, construção, } \\
\text { contextualização* }\end{array}$ & $\begin{array}{l}\text { Quality of Well-Being } \\
\text { Scale (OWS) }\end{array}$ & $\begin{array}{l}\text { Escores DRS } \\
\text { correlacionam-se com } \\
\text { OWB, com a subescala de } \\
\text { iniciação/perseveração } \\
\text { (função executiva), } \\
\text { apresentando maior } \\
\text { impacto }\end{array}$ & Sintomas depressivos \\
\hline Bankole et al. (2007)22 & $\begin{array}{l}198 \text { pacientes maiores } \\
\text { de } 55 \text { anos vivendo na } \\
\text { comunidade }\end{array}$ & $\begin{array}{l}\text { Atenção, memória, função } \\
\text { executiva, construção, } \\
\text { contextualização* }^{*}\end{array}$ & Quality of Life Index ${ }^{+}$ & $\begin{array}{l}\text { Melhor performance } \\
\text { cognitiva prediz pior QV } \\
\text { subjetiva }\end{array}$ & $\begin{array}{l}\text { Sintomas depressivos } \\
\text { e efeitos colaterais da } \\
\text { medicação }\end{array}$ \\
\hline
\end{tabular}


Tabela 1. Características dos artigos analisados (continuação)

\begin{tabular}{|c|c|c|c|c|c|}
\hline Estudo & População & Funções cognitivas & Medidas QV & Resultados & Outras associações \\
\hline Matsui et al. (2008)23 & $\begin{array}{l}53 \text { pacientes estáveis } \\
\text { vivendo na comunidade }\end{array}$ & $\begin{array}{l}\text { Função executiva e } \\
\text { memória }\end{array}$ & OLS\$ & Memória & Sintomas negativos \\
\hline Ristner (2007)24 & $\begin{array}{l}62 \text { pacientes estáveis } \\
\text { residindo na comunidade, } \\
\text { utilizando antipsicóticos } \\
\text { típicos (25), atípicos (25) e } \\
\text { combinaçãa dos dois (12) }\end{array}$ & $\begin{array}{l}\text { Habilidade motora, } \\
\text { atenção, memória, } \\
\text { aprendizado, atenção } \\
\text { sustentada e função } \\
\text { executiva }\end{array}$ & $\begin{array}{l}\text { - Quality of Life Scale } \\
\text { (OLS) } \\
\text { - Quality of Life } \\
\text { Enjoyment and Satisfation } \\
\text { Questionaire (Q-LES-O) }\end{array}$ & $\begin{array}{l}\text { Déficits em função } \\
\text { executiva, atenção visual } \\
\text { sustentada, memória } \\
\text { e habilidades motoras } \\
\text { explicam } 35 \% \text { na variação } \\
\text { das medidas da 00L\#\# }\end{array}$ & $\begin{array}{l}\text { Estresse emocional, } \\
\text { humor disfórico, } \\
\text { preocupações autísticas } \\
\text { (PANSS) e sintomas } \\
\text { positivos associam-se às } \\
\text { duas medidas de OV }\end{array}$ \\
\hline
\end{tabular}

* 0 estudo utilizou a Dementia Rating Scale (DRS) como medida cognitiva.

+ Escore da escala dado pelos próprios pacientes.

$\S$ Foram utilizados apenas sete itens da escala.

** Escore da escala dado pelos próprios pacientes.

\#\# A associação mantém-se significativa mesmo após controlar-se severidade de sintomas, efeitos colaterais, idade, educação e duração da doença.

\begin{tabular}{|c|c|c|c|c|c|}
\hline Williams et al. (2008)25 & $\begin{array}{l}56 \text { pacientes em primeiro } \\
\text { episódio em tratamento } \\
\text { ambulatorial }\end{array}$ & $\begin{array}{l}\text { Velocidade de } \\
\text { processamento de } \\
\text { informações, memória } \\
\text { verbal, memória de } \\
\text { trabalho, atenção, } \\
\text { função sensório-motora, } \\
\text { processamento verbal e } \\
\text { função executiva }\end{array}$ & WHOQOL-BREF & $\begin{array}{l}\text { Função executiva, } \\
\text { memória, memória } \\
\text { de trabalho, atenção, } \\
\text { velocidade de } \\
\text { processamento de } \\
\text { informações }\end{array}$ & $\begin{array}{l}0 \text { trabalho não analisou } \\
\text { a relação entre } \\
\text { sintomatologia e QV }\end{array}$ \\
\hline $\begin{array}{l}\text { Addington e Addington } \\
(2008)^{26}\end{array}$ & $\begin{array}{l}158 \text { indivíduos estudados } \\
\text { divididos em } 3 \text { grupos** }\end{array}$ & $\begin{array}{l}\text { Fluência verbal, memória } \\
\text { verbal e visual, memória } \\
\text { de trabalho, função } \\
\text { executiva, atenção, } \\
\text { habilidade de construção } \\
\text { visual, sequenciamento } \\
\text { visiomotor, velocidade } \\
\text { psicomotora }\end{array}$ & QLS\# & $\begin{array}{l}\text { Correlação entre cognição } \\
\text { e OLS }\end{array}$ & $\begin{array}{l}0 \text { trabalho não analisou } \\
\text { a relação entre } \\
\text { sintomatologia e QV }\end{array}$ \\
\hline Brissos et al. $(2008)^{27}$ & $\begin{array}{l}23 \text { pacientes estáveis em } \\
\text { tratamento ambulatorial. } \\
\text { Também inclui } 30 \\
\text { pacientes com transtorno } \\
\text { bipolar e } 23 \text { controles }\end{array}$ & $\begin{array}{l}\text { Atenção, habilidades } \\
\text { motoras, função } \\
\text { executiva, fluência verbal, } \\
\text { abstração verbal, atenção } \\
\text { visioespacial, memória }\end{array}$ & WHOQOL-BREF & $\begin{array}{l}\text { Não houve correlação } \\
\text { entre os quatro domínios } \\
\text { da escala e cognição }\end{array}$ & $\begin{array}{l}\text { Sintomas depressivos, } \\
\text { negativos e } \\
\text { psicopatologia geral } \\
\text { correlacionam-se a QV }\end{array}$ \\
\hline Narvaez et al. (2008)²8 & \begin{tabular}{|l}
88 pacientes estáveis \\
vivendo na comunidade
\end{tabular} & $\begin{array}{l}\text { Inteligência pré- } \\
\text { mórbida, velocidade } \\
\text { de processamento de } \\
\text { informações, memória } \\
\text { de trabalho, memória, } \\
\text { aprendizado, função } \\
\text { executiva }\end{array}$ & $\begin{array}{l}\text { Quality of Life Interview } \\
\text { (QOLI) }\end{array}$ & $\begin{array}{l}\text { Associação com medida } \\
\text { subjetiva da escala QV: } \\
\text { melhor performance } \\
\text { cognitiva prediz pior QV } \\
\text { subjetiva }\end{array}$ & $\begin{array}{l}\text { Sintomas depressivos } \\
\text { relacionam-se a QV } \\
\text { subjetiva e sintomas } \\
\text { negativos, a QV objetiva }\end{array}$ \\
\hline
\end{tabular}

* Composição dos grupos: $1^{0}$ - 50 pacientes em $1^{0}$ episódio psicótico; $2^{0}$ - 53 pacientes esquizofrênicos crônicos em tratamento ambulatorial; $3^{\circ}$ - 55 pessoas sem diagnóstico psiquiátrico. \# Foram também utilizadas a Social Functioning Scale e a Assessment of Interpersonal Problem Solving (AIPPS), uma medida de solução de problemas sociais.

\begin{tabular}{|c|c|c|c|c|c|}
\hline Perlick et al. (2008)29 & $\begin{array}{l}309 \text { pacientes com } \\
\text { diagnóstico de } \\
\text { esquizofrenia ou } \\
\text { transtorno esquizoafetivo, } \\
\text { a maioria vivendo na } \\
\text { comunidade }\end{array}$ & $\begin{array}{l}\text { Memória verbal, } \\
\text { coordenação motora, } \\
\text { sequenciamento visual, } \\
\text { fluência verbal e função } \\
\text { executiva }\end{array}$ & OLS & $\begin{array}{l}\text { Houve correlação entre } \\
\text { cognição e OV }\end{array}$ & $\begin{array}{l}\text { Sintomas foram mais } \\
\text { fortemente associados } \\
\text { a OV do que cognição, } \\
\text { explicando } 12 \% \text { da } \\
\text { variação no escore da } \\
\text { OLS contra } 8 \% \text { da função } \\
\text { cognitiva }\end{array}$ \\
\hline Mohamed et al. (2008)30 & $\begin{array}{l}1.386 \text { pacientes vivendo } \\
\text { na comunidade, crônicos }\end{array}$ & $\begin{array}{l}\text { Velocidade de } \\
\text { processamento, memória } \\
\text { verbal, atenção, função } \\
\text { executiva, raciocínio, } \\
\text { memória de trabalho }\end{array}$ & OLS & $\begin{array}{l}\text { Cognição foi significativa } \\
\text { e independentemente } \\
\text { associada ao escore da } \\
\text { OLS }\end{array}$ & $\begin{array}{l}\text { Sintomas explicam maior } \\
\text { variação no escore da OLS } \\
(15 \%) \text { do que cognição } \\
(6 \%)\end{array}$ \\
\hline Savilla et al. (2008)31 & $\begin{array}{l}57 \text { pacientes vivendo na } \\
\text { comunidade. Diagnóstico } \\
\text { confirmado por entrevista } \\
\text { estruturada }\end{array}$ & $\begin{array}{l}\text { Memória verbal, } \\
\text { memória de trabalho, } \\
\text { habilidade motora, } \\
\text { atenção e velocidade } \\
\text { de processamento de } \\
\text { informação, fluência } \\
\text { verbal, função executiva* }\end{array}$ & OLS & $\begin{array}{l}\text { Pior desempenho } \\
\text { cognitivo foi relacionado a } \\
\text { pior escore na OLS\# }\end{array}$ & $\begin{array}{l}\text { Sintomas também se } \\
\text { correlacionam a OLS }\end{array}$ \\
\hline
\end{tabular}

* Foi utilizada a Brief Assessment of Cognition in Schizophrenia (BACS).

\# Função executiva relacionou-se a todos os domínios da QLS, exceto Relações Interpessoais. Memória verbal e de trabalho e fluência verbal correlacionaram-se a funções intrapsíquicas. 
A população desses estudos é formada basicamente por pacientes esquizofrênicos adultos com transtorno crônico, estáveis, vivendo na comunidade e recebendo medicação antipsicótica típica a atípica. Exceções a esse perfil são os estudos de Mittal et al. ${ }^{21}$, Kasckow et al. ${ }^{10}$ e Bankole et al. ${ }^{22}$, que avaliaram populações com idade maior que 45 anos, e os estudos de Wegener et al. ${ }^{19}$, Addington e Addington ${ }^{26}$ e Williams et al. ${ }^{25}$, que estudaram uma população em primeiro surto psicótico. Já o estudo de Herman ${ }^{15}$ incluiu pacientes em tratamento hospitalar com abuso de substâncias associado.

A tabela 1 mostra o uso de vários tipos de escalas para avaliação da QV. Há um predomínio do uso da Quality of Life Scale (QLS) (10 estudos), da World Health Organization Quality of Life - Brief Form (WHOQOL-BREF) (6) e da Sickness Impact Profile (SIP) (3).

A QLS é uma escala de avaliação específica para esquizofrenia, e sua elaboração foi norteada pela síndrome deficitária do transtorno, objetivando avaliar os aspectos mais insidiosos da doença. A QLS possui uma estrutura multidimensional contendo quatro fatores e um total de 21 itens. Os fatores são relações interpessoais e rede social, papel ocupacional, funções intrapsíquicas e atividades e objetos comuns ${ }^{32}$.

A WHOQOL-BREF é um instrumento genérico de avaliação da $\mathrm{QV}$, desenvolvido para ser aplicado a pessoas que vivem sob diferentes circunstâncias, condições e culturas. A avaliação é feita pelos próprios pacientes e apresenta quatro domínios: físico, psicológico, relacionamentos sociais e fator ambiental. O domínio físico contém questões relacionadas a atividades diárias, adesão ao tratamento, dor e desconforto, sono e descanso, energia e fadiga. No domínio psicológico, há questões sobre sentimentos positivos e negativos, autoestima, imagem corporal e aparência física, crenças pessoais e atenção. O domínio de relacionamentos sociais está relacionado a relacionamentos pessoais, suporte social e atividade sexual. $\mathrm{O}$ domínio ambiental explora segurança física, recursos financeiros, cuidados sociais e de saúde e sua disponibilidade, oportunidades para aquisição de novas informações e habilidades e participação e oportunidades para recreação e transporte14,18,19.

A SIP é um instrumento de avaliação subjetiva da qualidade de vida feita pelos próprios pacientes. Foi desenvolvida para medir disfunção relacionada à doença, independente da presença objetiva desta e da experiência subjetiva de desconforto relacionado à doença. A escala original possui 136 questões representando 12 categorias de QV. Nos artigos analisados, entretanto, foram utilizadas somente categorias relevantes às disfunções causadas pela esquizofrenia, como interação social, comunicação, sono e repouso, recreação e cuidados com o lar ${ }^{2,12,16}$.

De forma geral, os domínios cognitivos mais estudados foram: função executiva, memória (verbal e visual), atenção, fluência verbal e memória de trabalho. Alguns estudos também incluíram em sua avaliação habilidades visuais e verbais, velocidade motora e inteligência.

Há grande variabilidade nos testes neuropsicológicos utilizados para avaliação das funções cognitivas. Os testes mais usados para avaliação dos principais domínios cognitivos estão apresentados na tabela 2 .

Tabela 2. Funções cognitivas e testes neuropsicológicos mais utilizados

\begin{tabular}{l|l}
\hline Função cognitiva & \multicolumn{1}{c}{ Testes neuropsicológicos } \\
\hline Função executiva & $\begin{array}{l}\text { Wisconsin Sorting Card Test (WCST), Trail Making } \\
\text { Part Be testes de fluência verbal }\end{array}$ \\
\hline Memória & $\begin{array}{l}\text { Weschsler Memory Scale, Evocação de lista de } \\
\text { palavras e Teste da Figura Complexa de Rey }\end{array}$ \\
\hline Atenção & Digit Span Test e Continuous Performance Test \\
\hline Fluência & Controlled Oral Word Association Test \\
\hline Memória de trabalho & Digit Span Test \\
\hline
\end{tabular}

\section{Associação entre déficit cognitivo e QV}

Dos 27 estudos analisados, 20 (74,1\%) encontraram a associação entre déficits cognitivos e QV. Nestes, a associação foi leve ou moderada. No estudo de Addington e Addington ${ }^{8}$, a relação deixa de ser significativa após levar-se em conta a sintomatologia negativa. Os autores salientam que a população desse estudo foi constituída por pacientes de alto desempenho cognitivo, o que pode justificar por que a associação entre cognição e qualidade de vida não apresentou significância. Já no estudo de Kasckow et al. ${ }^{10}$, a medida da função cognitiva utilizada foi o Mini-Mental, que foi preditor do escore da escala de QV após seis meses de seguimento.

A associação entre função cognitiva e QV foi evidenciada mais em estudos com escalas respondidas pelos pesquisadores do que nos estudos com escalas respondidas pelos próprios pacientes (de autoavaliação). Dentre os primeiros (12 estudos 6,8,10,17,20-22,24,26,29-31), apenas Addington e Addington ${ }^{8}$ não encontraram associação, já que esta desapareceu ao se considerar o efeito da sintomatologia. Assim, ocorreu associação entre QV e cognição em 91,7\% dos estudos que utilizaram escalas respondidas pelos pesquisadores. Já dentre os estudos que utilizam autoavaliações de QV, 912-16,19,22,25,28 entre 152,7,9,11-16,18,19,22,25,27,28 estudos (60,0\%) encontraram associações. Em dois deles 22,28 , a associação foi paradoxal, já que melhor função cognitiva foi preditora de pior QV.

Dentre os estudos que analisaram separadamente os domínios cognitivos, percebe-se maior associação entre QV e função executiva (11 em 13 estudos), memória (8 em 10 estudos), atenção (6 em 7 estudos), fluência ( 3 em 3 estudos), habilidades motoras ( 2 em 2 estudos) e memória de trabalho (3 em 5 estudos).

\section{Discussão}

Os resultados dessa revisão indicam importante associação entre déficits cognitivos e QV em pacientes portadores de esquizofrenia. Do total de 27 estudos, em 20 a associação foi significativa. Esses resultados assemelham-se à associação entre cognição e funcionamento na comunidade e atividades diárias verificada nesses pacientes na metanálise de Green et al. ${ }^{33}$. A relação entre cognição e QV, contudo, apresenta peculiaridades que precisam ser consideradas. Os estudos sobre o tema agrupam-se em dois tipos principais. No primeiro, a QV é avaliada por observadores/pesquisadores e é considerada uma medida mais objetiva que o segundo tipo. Neste são usadas escalas de autoavaliação em que os pacientes dão escores sobre sua percepção de QV, sendo, portanto, uma medida mais subjetiva. Os resultados encontrados mostram que a associação com déficits cognitivos é mais importante entre as medidas de QV feitas por observadores comparadas às medidas de autoavaliação, ao menos em números absolutos. Apenas 1 entre 12 estudos não encontrou associação entre QV avaliada por observador e funções cognitivas, ao passo que, em 9 entre 15 estudos que se utilizaram de autoavaliações dos pacientes, a associação não foi encontrada. Os estudos de Narvaez et al. ${ }^{28}$ e Bankole et al. ${ }^{22}$ demonstraram relações paradoxais entre QV e função cognitiva, ou seja, a medida de QV subjetiva foi melhor em pacientes com pior desempenho cognitivo.

Tal relação paradoxal pode se relacionar a fatores que afetam a percepção e as expectativas dos pacientes. Como o julgamento dos pacientes com esquizofrenia geralmente está comprometido, sobretudo naqueles com déficit cognitivo pronunciado, viver em condições que parecem adversas e desagradáveis, para clínicos e avaliadores, pode não se traduzir em pior satisfação com a vida ${ }^{4}$. Expectativas em relação à vida estão geralmente diminuídas na esquizofrenia quando os pacientes se acomodam às circunstâncias adversas. Por outro lado, no contexto de uma intervenção terapêutica positiva ${ }^{34} \mathrm{O}$ aumento da expectativa pode levar a uma queda paradoxal na QV subjetiva. Assim, pacientes com melhor desempenho cognitivo, que reconhecem seus déficits, podem apresentar pior QV em relação àqueles mais empobrecidos, que apresentam insight pobre ${ }^{35}$. 
Nesse sentido, Brekke et al. ${ }^{36}$ demonstraram que pacientes com função executiva preservada não mostraram maior satisfação com a vida quando apresentaram melhorias em indicadores psicossociais. Os autores sugerem que funções executivas como abstração e consciência do contexto ambiental permitem perceber que o ganho adquirido ainda é muito pequeno se comparado ao restante da comunidade. Já nos pacientes com disfunção executiva, a falta dessa percepção faz com que qualquer ganho psicossocial resulte em melhor satisfação com a vida.

Os resultados da presente revisão corroboram vários estudos que demonstram que os conceitos de QV objetiva e subjetiva podem ser construtos diferentes, em virtude das disparidades vistas entre avaliações feitas por observadores e autoavaliações realizadas pelos próprios pacientes. Um grande número de estudos que avaliaram a QV com ambos os métodos falhou em encontrar uma relação entre eles. Os estudos que examinaram os determinantes das duas formas de avaliação da QV também encontraram disparidades ${ }^{37,38}$.

A falta de relação entre a percepção da QV pelo próprio paciente (subjetiva) e a QV medida por observador (objetiva) não indica que informação proveniente da percepção do paciente deva ser descartada, pois é falha. O conceito de QV sempre abarca um aspecto subjetivo, que deve ser levado em consideração. Assim, as medidas subjetivas e objetivas devem ser consideradas como informações complementares sobre o paciente em questão ${ }^{33}$, já que provavelmente sofrem influência de fatores distintos e independentes.

A medida de QV, portanto, é profundamente alterada pelo instrumento e os informantes utilizados para acessá-la. A escolha de instrumentos para avaliação da QV deve ser feita com claro entendimento de qual informação se quer obter e os resultados devem ser relatados com clara explicação metodológica ${ }^{33}$. Em pacientes com prejuízo cognitivo acentuado, medidas de QV avaliadas por observadores podem ser mais úteis por serem menos afetadas por esse prejuízo. Por outro lado, pacientes com melhor desempenho cognitivo provavelmente apresentam maior credibilidade em suas avaliações subjetivas de QV, podendo, assim, ser a avaliação das funções cognitivas um fator que pode conferir mais validade às autoavaliações ${ }^{3}$.

Este estudo é limitado pela não inclusão de artigos cuja avaliação de QV estava relacionada a ensaios farmacológicos ou faziam parte da avaliação de programas de reabilitação. Isso foi feito por se acreditar que o perfil cognitivo e de QV dessas populações seletas pode diferir do dos demais pacientes. O pequeno número de artigos com essas características ainda não permite uma análise específica dessas populações. Assim, mais estudos com essas populações são necessários para confirmar se a análise feita aqui pode ser extrapolada para seus pacientes.

\section{Conclusão}

Os estudos sobre cognição e QV, em sua maioria, mostram correlação significativa em pacientes com esquizofrenia. Essa associação é mais evidente nos estudos que utilizaram medidas de QV feitas por pesquisadores do que naqueles que utilizaram autoavaliações dos pacientes. Essa diferença pode se relacionar ao prejuízo na capacidade avaliativa dos pacientes, a qual está relacionada justamente ao prejuízo cognitivo. Assim, a avaliação cognitiva é importante não somente como parâmetro determinante da QV dos pacientes, mas também por auxiliar na interpretação das escalas de QV, de modo que as escalas por autoavaliações parecem mais adequadas em pacientes com menor prejuízo cognitivo.

\section{Referências}

1. Sharma T, Antonova L. Cognitive function in schizophrenia. Deficits, functional consequences and future treatment. Psychiatr Clin N Am. 2003;26:25-40.

2. Heslegrave RJ, Awad AG, Voruganti LNP. The influence of neurocognitive deficits and symptoms on quality of life in schizophrenia. J Psychiatry Neurosci. 1997;22:235-43.

3. Voruganti LNP, Heslegrave RJ, Awad AG. Quality of life measurement during antipsychotic drug therapy of schizophrenia. J Psychiatry Neurosci. 1997;22:267-74.
4. Fleck MPA, Leal OF, Louzada S, Xavier M, Chachamovich E, Vieira G et al. Desenvolvimento da versão em português do instrumento de avaliação da qualidade de vida da OMS (WHOQOL-100). Rev Bras Psiquiatr. 1990;21:19-28

5. Priebe S. Social outcomes in schizophrenia. Br J Psychiatry. 2007;191(50):15-20.

6. Meltzer HY, Thompson PA, Lee MA, Ranjan R. Neuropsychologic deficits in schizophrenia: relation to social function and effect of antipsychotic drug treatment. Neuropsychopharmacology. 1996;14:27-33.

7. Dickerson FB, Ringel NB, Parente F. Subjective quality of life in outpatients with schizophrenia: clinical and utilization correlates. Acta Psychiatr Scand. 1998;98:124-7.

8. Addington J, Addington D. Neurocognitive and social functioning in schizophrenia. Schizophr Bull. 1999;25(1):173-82.

9. Smith TE, Hull J, Goodman M, Hedayat-Harris A, Willson DF, Israel L, et al. The relative influence of symptoms, insight and neurocognition on social adjustment in schizophrenia and schizoaffective disorder. J Nerv Ment Dis. 1999;187:102-8.

10. Kasckow JW, Twamley E, Mulchahey JJ, Carroll B, Sabai M, Strakowski $\mathrm{SM}$, et al. Health-related quality of well-being in chronically hospitalized patients with schizophrenia: comparison with matched outpatients. Psychiatry Res. 2001;103:69-78.

11. Aksaray G, Oflu S, Kaptanoglu C, Bal C. Neurocognitive deficits and quality of life in outpatients with schizophrenia. Prog Neuropsychopharmacol Biol Psychiatry. 2002;26:1217-9.

12. Vaz SAM, Heinrichs RW. Schizophrenia and memory impairment: evidence for a neurocognitive subtype. Psychiatry Res. 2002;113:93-105.

13. Fujii DE, Wylie AM, Nathan JH. Neurocognition and long-term prediction of quality of life in outpatients with severe and persistent mental illness. Schizophr Res. 2004;69:67-73.

14. Alptekin K, Akvardar Y, Akdede BBK, Dumlu K, Isik D, Pirinçci F, et al. Is quality of life associated with cognitive impairment in schizophrenia? Prog Neuropsychopharmacol Biol Psychiatry. 2004;29:239-44.

15. Herman M. Neurocognitive functioning and quality of life among dually diagnosed and non-substance abusing schizophrenia inpatients. Int J Ment Health Nurs. 2004;13:282-91.

16. Sota TL, Heinrichs RW. Demographic, clinical and neurocognitive predictors of quality of life in schizophrenia patients receiving conventional neuroleptics. Compr Psychiatry. 2004;45:415-21.

17. Cavieres AF, Valdebenito MV. Funcionamiento cognitivo y calidad de vida en la esquizofrenia. Rev Chil Neuropsiquiatr. 2005;43:97-108.

18. Hofer A, Baumgartner S, Bodner T, Edlinger M, Hummer M, Kemmler G, et al. Patient outcomes in schizophrenia II: the impact of cognition. Eur Psychiatry. 2005;20:395-402.

19. Wegener S, Redoblado-Hodge MA, Lucas S, Fitzgerald D, Harris A Brennan J. Relative contributions of psychiatric symptoms and neuropsychological functioning to quality of life in first-episode psychosis. Aust N Z J Psychiatry. 2005;39:487-92.

20. Bozikas VS, Kosmidis MH, Kafantari A, Gamvrula K, Vasiliadou E, Petrikis P, et al. Community dysfunction in schizophrenia: rate-limiting factors. Prog Neuropsychopharmacol Biol Psychiatry. 2006;30:463-70.

21. Mittal D, Davis CE, Depp C, Pyne JM, Golshan S, Patterson TL, et al. Correlates of health-related quality or well-being in older patients with schizophrenia. J Nerv Ment Dis. 2006;194:335-40.

22. Bankole AO, Cohen CI, Vahia I, Diwan S, Kehn M, Ramirez PM. Factors affecting quality of life in a multiracial sample of older persons with schizophrenia. Am J Geriatr Psychiatry 2007;15:1015-23

23. Matsui M, Sumiyoshi T, Arai H, Higuchi Y, Kurachi M. Cognitive functioning related to quality of life in schizophrenia. Prog Neuropsychopharmacol Biol Psychiatry. 2008;32:280-7.

24. Ristner MS. Predicting quality of life impairment in chronic schizophrenia from cognitive variables. Qual Life Res. 2007;16:929-37.

25. Williams LM, Whitford TJ, Flynn G, Wong W, Liddell BJ, Silverstein S. General and social cognition in first episode schizophrenia: identification of separable factors and prediction of functional outcome using the IntegNeuro test battery. Schizophr Res. 2008;99:182-91.

26. Addington J, Addington D. Social and cognitive functioning in psychosis. Schizophr Res. 2008;99:176-81.

27. Brissos S, Dias VV, Carita AI, Martinez-Arán A. Quality of life in bipolar type I disorder and schizophrenia in remission: clinical and neurocognitive correlates. Psychiatry Res. 2008;160:55-62. 
28. Narvaez JN, Twamley EW, McKibbin CL, Heaton RK, Patterson TL Subjective and objective quality of life in schizophrenia. Schizophr Res. 2008;98:201-8.

29. Perlick DA, Rosenheck RA, Kaczynski R, Bingham S, Collins J. Association of symptomatology and cognitive deficits to functional capacity in schizophrenia. Schizophr Res. 2008;99:192-9.

30. Mohamed S, Rosenheck R, Swartz M, Stroup S, Lieberman JA, et al. Relationship of cognition and psychopathology to functional impairment in schizophrenia. Am J Psychiatry. 2008;165:978-87.

31. Savilla K, Kettler L, Galletly C. Relationship between cognitive deficits, symptoms and quality of life in schizophrenia. Aust N Z J Psychiatry. 2008;42:496-504.

32. Cardoso CS, Bandeira M, Caiaffa WT, Fonseca JOP. Escala da qualidade de vida para pacientes com esquizofrenia (QLS-BR): adaptação transcultural para o Brasil. J Bras Psiquiatr. 2002;51(1):31-8.

33. Green MF, Kern RS, Braff DL, Mintz J. Neurocognitive deficits and functional outcome in schizophrenia: are we measuring the "right stuff"? Schizophr Bull. 2000;26:119-36.
34. Pinikahana J, Happell B, Hope J, Keks NA. Quality of life in schizophrenia: a review of the literature from 1995 to 2000. Int J Ment Health Nurs. 2002;11:103-11.

35. Atkinson $\mathrm{M}$, Zibin $\mathrm{S}$, Chuang $\mathrm{H}$. Characterizing quality of life among patients with chronic mental illness: a critical examination of the selfreport methodology. Am J Psychiatry. 1997;154:99-105.

36. Brekke JS, Kohrt B, Green MF. Neuropsychological functioning as a moderator of the relationship between psychosocial functioning and the subjective experience of self and life in schizophrenia. Schizophr Bull. 2001;24:697-708.

37. Fitzgerald PB, De Castella ARA, Filia K, Collins J, Brewer K, Williams $\mathrm{CL}$, et al. A longitudinal study of patient- and observer-rated quality of life in schizophrenia. Psychiatry Res. 2003;119:55-62.

38. Kusel Y, Laugharne R, Perrington S, McKendrick J, Stephenson D, Stockton-Henderson J, et al. Measurement of quality of life in schizophrenia: a comparison of two scales. Soc Psychiatry Psychiatr Epidemiol. 2007;42:819-23. 